\title{
Rasgos de la práctica de la administración en la civilización agrícola, la industrialización y la cibereconomia en mercados globales
}

\author{
Features of the practice of the administration in agricultural civilization, industrialization and \\ cybereconomy in the global markets
}

Resumen: Este artículo responde la pregunta: ¿Qué une a las actividades de la práctica de la administración del siglo XXI con aquellas de los grupos considerados "primitivos"?: similitudes en armar equipo de trabajo; comportamientos, objetivos y asimetrías por diferencias en modos de producción. Objetivos: (1) Rescatar del trabajo de grupo de la comunidad "primitiva" Gouro la solidaridad, el apoyo mutuo y la coordinación de prácticas económicas, requeridas en la implementación de estrategias de ruptura en organizaciones amenazadas por la competencia de mercados saturados y gestión interna basada en el individualismo y realización personal. (2) Mostrar la pertinencia de la interdisciplinariedad como aporte a la alta gerencia en identificación de problemas y alternativas de respuesta. Las amenazas ambientales del entorno obligan a emprender estudios interdisciplinarios para redefinir tendencias de prospectiva según el área que requiera resolverse desde la práctica de la administración. Categorías: espacio/tiempo, división social del trabajo; liderazgo, trabajo de equipo y valores. La metodología es de síntesis.

Palabras clave: Agricultura; Industrialización; Cibereconomía; División técnica del trabajo; Trabajo de equipo.

JEL: M 14, M4

Abstract: This article answers the question that assails the administrator: What unites the activities of the management practice of the XXI century with those of groups considered "primitive"?: Similarities build teamwork and asymmetries similar behaviors and other behaviors by differences in production methods. Objectives: (1) Rescue work group of "primitive" Gouro community solidarity, mutual support and flawless coordination of economic practices, which in turn are required in the implementation of strategies for breaking into organizations threatened by competition saturated markets and internal management based on individualism and personal fulfillment. (2) Shows the relevance of interdisciplinarity as an input to senior management in identifying problems and possible answers. Environmental threats in the environment force them to undertake interdisciplinary studies prospectively to redefine trends by area requiring resolved from the practice of management. Categories: space/ time, social division of labor; leadership, teamwork and values. The synthesis methodology.

Keywords: Agriculture; Industrialization; Cybereconomy; Technical division of labor; Teamwork.

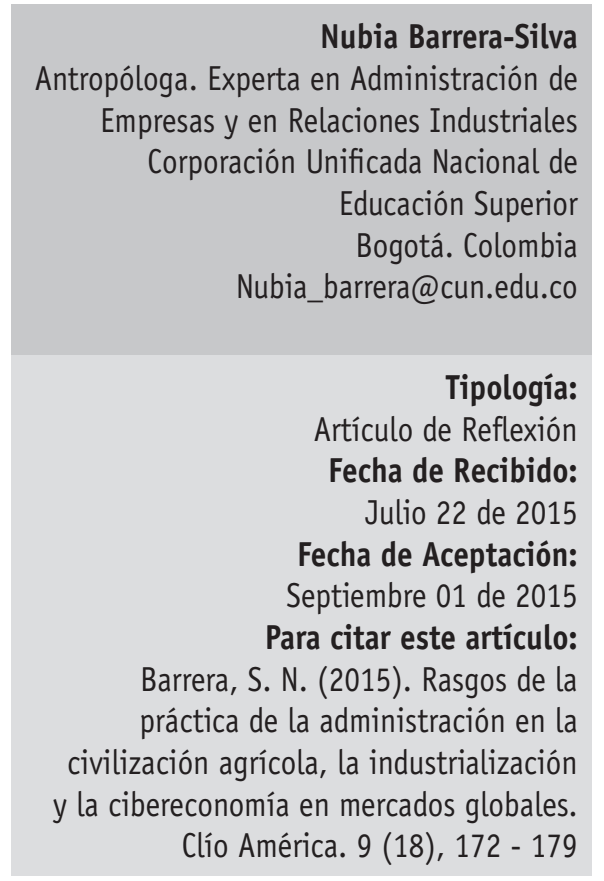




\section{Introducción}

Se identifican rasgos relevantes de la práctica de la administración de los modos de producción de la agricultura, la industrialización pasando por la comunidad Gouro y la cibereconomía. La investigación etnográfica de Meillaseaux demuestra que la práctica de la administración está inmersa en la coordinación del trabajo de grupo, aunque el concepto no existiese como tal. De igual manera, Bijon analiza la concepción del cambio, la solidaridad y la pertenencia a la organización. "La cultura moviliza los esfuerzos individuales porque induce una manera de ser y de actuar en la empresas. Vivifica y federa los equipos en enfoques comunes de la realidad" (1992, p. 197).

Asimismo, se mencionan aportes de Taylor y Fayol en los inicios de la disciplina administrativa como ciencia. Finalmente, en la ciberconomía y marketplaces se enfatiza en la deslocalización de mercados saturados y la inminente necesidad de construir estrategias y escenarios según el tamaño de las organizaciones, la misión y visión para comprender la dinámica del mercado global en las localidades y la región. Aunque se mantiene la división técnica del trabajo en procesos avanzados de producción -sin que sea el objetivo de este artículo-, la globalización trae hechos inéditos con respecto a modos de producción anteriores, que exige a los líderes diferentes perspectivas de visión de futuro.

Finalmente, Ferris (1988) resume el propósito central de este artículo en la intención de recurrir a momentos de la historia de la práctica de la administración cuando afirma: "(...) la historia, según dicen, se comprende en retrospectiva, aunque tiene que vivirse hacia el futuro, y cuando estudiamos a nuestros predecesores, llevamos nuestras propias lámparas" (p. 3).

\section{Ideas generales de la interdisciplinariedad en la práctica de la administración}

La pregunta que asalta al administrador: ¿Qué une a la práctica de la administración del siglo XXI con aquella de los grupos humanos considerados "primitivos"?
El capitalismo financiero, la globalización de los medios de comunicación, la cibereconomía y el cambio climático - por señalar algunos de los eventos inéditos de más significación histórica actual-, replantean la pertinencia del objeto de estudio de cada disciplina en las ciencias, en el sentido de percibir y comprender mejor los conflictos socioeconómicos y culturales que aquejan a las organizaciones en entornos amenazantes en perspectiva global. Con frecuencia se invoca la famosa frase de Napoleón Bonaparte: "Quien no conoce su historia, está condenada a repetirla" sin que se comprenda su verdadero significado, sobre todo en países que desdeñan o restan importancia a su propio quehacer en la historia. Por su parte, Ferris (1988) menciona que:

Henry Ford creía que la historia era 'más o menos tonterías' y que la única historia que importaba era la que hacemos en el presente (...) Burke (1978) lo expresa mejor cuando pregunta por qué debemos mirar al pasado a fin de prepararnos para el futuro. La respuesta es sencilla: 'porque no hay otra parte hacia dónde mirar'. (p. 3)

Coincidiendo con Burke (citado por Ferris 1988), el enfoque de momentos de la historia pretende crear espacios de encuentro entre la administración y la antropología cultural, dos áreas disciplinares que construyen conocimiento desde dos grandes categorías: la cultura organizacional con formas diferentes de hacer las cosas con fines de productividad y generación de ganancias.

En este orden de ideas, los cambios de denominación de grupos de trabajo o de equipos de trabajo corresponden a los modos de producción de las épocas estudiadas. En los grupos considerados "primitivos" y actuales de pueblos étnicos corresponden al carácter de propiedad colectiva (resguardo) basada en relaciones sociales de apoyo mutuo; en tanto que, los equipos de trabajo en las organizaciones del siglo XXI se sustentan en la propiedad privada, el individualismo y la competencia tanto del mercado como de las personas en las organizaciones. En este sentido, la historia aporta ideas de culturas diferentes para armar estrategias de gerencia basadas en la solidaridad, cooperación y la conciencia del espíritu 
Rasgos de la práctica de la administración en la civilización agrícola, la industrialización y la cibereconomía en mercados globales

colectivo que requiere el cambio relacionado con la visión empresarial.

La cibereconomía y el surgimiento de nuevas formas de ciudadanía con impacto en los mercados globales, complejizan de tal manera los procesos de gestión y resolución de problemas administrativos, que resulta pertinente acudir a la interdisciplinariedad entendida como exigencia interna de la práctica de la administración e interacción entre disciplinas que conminan trascender la propia especificidad del área del conocimiento (Tamayo, 1995).

\section{De la agricultura a la industrialización pasando por la comunidad "primitiva" GOURO}

Si bien el periodo de la revolución industrial significó dejar atrás el predomino del modo de producción agrícola anclada en la familia extensa, esta unidad económica exigió un trabajo colectivo con distintas formas de ayuda mutua "vinculadas con estructuras de dirección y de control que son el efecto común de las exigencias técnicas del proceso y de las relaciones de producción en las que se lleva a cabo ese proceso" (Terray, 1971, p. 101).

No sobra advertir que, en las relaciones de producción se gestan las relaciones de distribución y que a su vez inciden en la definición de unidades de consumo. Esta secuencia dialéctica recorrió las mentes de los individuos de la industrialización como de las instituciones económicas, sociales y culturales que garantizaban la eficacia y eficiencia del sistema agrícola basado en la explotación de fuentes renovables de energía humana y de animales que surcaban la tierra. El tiempo transcurría al ritmo de la siembra, crecimiento de cultivos, cosecha, almacenaje y distribución de alimentos tanto en el mercado como entre los integrantes de la familia extensa. Los cambios de luz solar del transcurso del día y lapsos de tiempo destinados a las actividades cotidianas como rezar un padrenuestro o cocinar alimentos definían la duración del tiempo y horas destinadas a la faena diaria. Por la etnografía y la historia sabemos que este fenómeno se repitió sin cesar en confines distantes de la tierra.
De las entrañas de la agricultura surge frenéticamente la revolución industrial, ésta expulsó de las faenas agrícolas a hombres, mujeres y niños para concentrarlos en barracas una vez terminaban las extensas jornadas de trabajo. Las actividades cotidianas de la familia extensa y labores del campo, dieron paso al sonido de la campana que anunciaba el ingreso a la factoría.

Tofler afirma que el periodo industrial "reunió varias máquinas en sistemas interconectados y bajo un mismo techo, creando la factoría y, finalmente, la cadena de montaje dentro de la factoría" (1981, p. 41). A las grandes transformaciones las denominó indusrealidad por la creación de nuevas matrices de mercado con base en el dinero; la separación de la producción del consumo anclada en (i) la uniformización afirmada en la producción en serie y demanda asegurada; fundación de industrias y procedimientos comerciales y de contratación, el desarrollo de la administración con base en productos y procesos, creación de pesos y medidas, el trabajo y escalas de salarios que abarcó sinnúmero de campos de la vida material de las sociedades; (ii) la especialización preconizada por Taylor surgió como efecto de la división del trabajo; (iii) la sincronización separó la producción del consumo y definió el tiempo un equivalente del dinero; (iv) la concentración transformó la vida de las personas en las ciudades, grandes capitales se sumergieron en la corporación gigante y el monopolio; las industrias de alimentos, automóviles, rollos fotográficos, tintes y pinturas se concentraron en grandes compañías; (v) la maximización expresada en un caso de "marcofilia" por las grandes dimensiones, el crecimiento y el desarrollo: el rascacielos más alto, el embalse más grande, aumentar la producción y exportar a los pueblos del mundo.

Agregando a lo anterior, los medios de comunicación entregaron a la gente la visión del mundo que el capitalismo requería: la supremacía de la tecnología forjada en la producción en serie a costa de la destrucción de los recursos naturales, crisis en las instituciones sociales, políticas y culturales; pobreza generalizada a pesar de avances significativos en servicios colectivos. En resumen, la hegemonía del 
mercado consolidó el sistema capitalista en detrimento de la unidad de la naturaleza.

Al llegar aquí tenemos que, la conexión irrestricta entre el capital y el trabajo asalariado exigió productividad máxima que en ese tiempo recaía casi exclusivamente en el trabajador, por ser el ejecutor directo del proceso en la cadena de montaje, Haber (1964) estableció que:

Era deber del gerente compilar, tabular, clasificar y, por medio de la aplicación de la ciencia, reducir esta información a principios, reglas, leyes y fórmulas que resumieran todas las experiencias de los trabajadores y pudieran utilizarse como lineamientos para desempeñar mejor las tareas cotidianas. (p. 58)

De acuerdo a lo anterior, el pensamiento de Taylor (citado por Verdugo, 2010) se resume en "(...) lograr el hombre idóneo para cada función y proporcionarle el equipo adecuado y una organización eficiente" ( $p$. 21). Desde este enfoque, investigó in situ los tiempos/movimientos del obrero en trabajos de rutina productiva adjunto a la máquina, con el propósito de eliminar movimientos innecesarios que disminuyesen en tiempo real el número de operaciones para terminar una unidad. Tofler resume con base en los "modelos T" que fabricó Ford en 1908, que "el trabajo especializado requería, no una persona completa, sino solo una parte. Nunca se ha aducido una prueba más vívida de que la superespecialización puede resultar embrutecedora" (1981, p. 62).

\section{El trabajo de grupo desde la historia y en perspectiva interdisciplinaria}

En la época de Taylor, la investigación con métodos científicos en la práctica de la administración irrumpió como campo del conocimiento en las ciencias sociales. De otra parte, antes de que existiese la necesidad de abordar la práctica social desde la interdisciplinariedad, cada área y subdivisión especializada seleccionaba y reconstruía desde la historia hechos 0 acontecimientos sociales que parecían hacer parte de la disciplina involucrada.

Ahora bien, con la globalización no solo se descubren nuevas fronteras del conocimiento sino que aparecen interpretaciones y conceptos diferentes de distintas disciplinas sobre un mismo hecho 0 práctica social. Por ende, se requieren nuevos abordajes del conocimiento para descubrir pertinencias, aportes, coincidencias o diferencias conceptuales en la comprensión de procesos y aplicaciones concretas en resolución de problemas inéditos que inciden directamente en la eficiencia de la administración. Para Solano (2007) "significa ver un problema desde diversas perspectivas, pero en una forma consciente e interrelacionada. No es un esquema fácil si se desea tener resultados, porque implica poner de acuerdo las diversas percepciones de vida que tiene cada tipo de profesional" (p. 2). En este caso, la antropología cultural y la práctica de la administración se acercan al trabajo en grupo en una comunidad "primitiva" con respecto al de sociedades avanzadas en 0ccidente.

Regresando al trabajo complejo en las comunidades "primitivas" desde tiempo inmemorable, meillassoux (citado por Terray, 1971), analiza la conformación de equipos reclutados entre vecinos del iniciador de la empresa en forma momentánea e intermitente, que arrojaba un producto instantáneo y exigía cooperación discontinua y provisional en la caza de animales con red. El número de hombres que participaba correspondía al tamaño, la especie de animal y estrategias de caza en el territorio. De análoga manera, en las instituciones actuales prevalece el trabajo de equipo: sus integrantes elaboran conductas similares en situaciones de emergencia ambiental (inundaciones, heladas, sismos entre otros) 0 en ejecución de proyectos de desarrollo en las comunidades. Las instituciones arman grupos temporales 0 a largo plazo, según las metas propuestas en zonas estratégicas y una vez finalizada la emergencia o la ejecución del proyecto, desaparece el grupo.

En este orden de ideas, las organizaciones inmersas en mercados globales necesitan redefinir sus estrategias de gerencia con base en la cooperación entre el equipo de base y gerentes de línea en aras del cumplimiento de sus propias metas. Bijon (1992) sostiene que: "Es imposible organizar a las personas que tienen que vivir juntas en medio de una competencia permanente. La regla interna solo puede ser la superación: dar a cada cual la oportunidad de expresarse y superarse" (p. 96). 
Rasgos de la práctica de la administración en la civilización agrícola, la industrialización y la cibereconomía en mercados globales

\section{La ciencia administrativa entre la práctica y la fundamentación teórica}

Se atribuye a los ingenieros la creación del movimiento de la administración científica, sus integrantes construyeron buena parte de la teoría y la práctica moderna, casi al punto de llamarse administración de la eficiencia vinculada a los valores. Mencionar la administración como área de la ciencia es remitirse de inmediato a Taylor. Así pues, Haber (citado por Duncan, 1999) piensa que:

Los propósitos que impulsaron a Taylor a escribir The Principes of Scientific Management (1914) fueron 1. Demostrar, mediante ilustraciones sencillas, las pérdidas que sufría Estados Unidos de América por falta de eficiencia. 2. Convencer al lector de que la solución a dicha pérdida se encuentra en la administración sistemática y no en la búsqueda inútil de trabajadores extraordinarios. 3. Probar que la mejor administración es una verdadera ciencia que se basa en leyes, reglas y principios definidos. 4. Probar también que los principios de la administración científica son aplicables a todas las actividades humanas $y$, cuando se usan correctamente, producen resultados asombrosos. (p. 57)

Con respecto al segundo punto, Taylor ampliamente demostró que su aporte a la teoría administrativa consistía en uniformizar tareas en el puesto de trabajo con desarrollo de actividades mecánicas y repetitivas, soporte de la sincronización de tiempos y movimientos con fines de máxima productividad por parte del trabajador para alcanzar "resultados asombrosos".

Se subraya que la aplicación de principios administrativos permite enlaces de intereses entre propietarios de medios de producción y trabajadores, donde la "prosperidad" beneficia más que todo a aquel; mientras que el trabajador medio apenas deriva satisfactores de subsistencia. Sin embargo, ninguno puede existir sin la participación del otro. Para Marx, el propietario de los medios de producción posee el capital y el salario de los trabajadores; por lo tanto, dispone del poder y la autoridad que exige la subordinación del trabajador al dueño de los medios de producción capitalista.
Siguen los aportes de Henry Fayol (1841-1925), Ingeniero de minas. Su experiencia se encaminó hacia la funcionalidad de las empresas comerciales: "Aisló sus observaciones sobre las actividades que realizan los gerentes, la importancia de las habilidades administrativas en relación con la pericia técnica, y algunos principios fundamentales de la administración" (Schwartz, 1999, p. 27). Estableció seis tipos de actividades: técnicas, comerciales, financieras, seguridad, contabilidad y las actividades directivas.

Conviene resaltar en las operaciones administrativas las siguientes actividades: planear, organizar, coordinar, ejercer el mando y controlar, todavía parecen procedimientos insustituibles en las modernas teorías administrativas y gerenciales. Aunque no es aquí el lugar para ampliar el contenido de las operaciones administrativas, sí sobresalen tres aspectos importantes: (a) se encuentran en las actividades de las comunidades que antecedieron a la industrialización acorde al desarrollo técnico y tecnológico de sus respectivos momentos en la historia con otro lenguaje y formas de hacer las cosas; (b) aclaran las funciones del rol directivo de gerentes, líderes 0 coordinadores; y (c) complementan desde la parte interna del proceso administrativo los aportes de Taylor. Desde la investigación, el rol de la empresa es un tema central. Es más que un agente económico en la economía del mercado. Es también el lugar donde se reúnen grupos de personas y siguen estrategias previamente concebidas que permitan a las organizaciones alcanzar sus objetivos. En este sentido, resulta valioso mencionar los aportes:

De las experiencias y teorías de los años 19201935 en los Estados Unidos: reforma a la gestión de la General Motors, las teorías de Taylor, Mac Gregor y las experiencias psicosociológicas (Mayo y Hawthorne) aplicadas a la organización en talleres, lugares que destinaban los trabajadores para determinar las tareas de cada uno; ascensos, rotaciones y ritmos y cadencias del trabajo. (Anónimo, 1974, pp. 35-36) Traducción propia.

Siguiendo con la analogía de la práctica de la administración, Fayol define la actividad administrativa por un conjunto de acciones vinculadas a 
la previsión, organización, mando, coordinación y control. Las diferencias entre 0ccidente de economía capitalista y las comunidades "primitivas" son el modelo económico y el contexto en que se realizan estas funciones en la empresa, el grado de desarrollo de técnicas, los medios de trabajo y las formas de cooperación restringida o ampliada que generan las actividades económicas con las técnicas de cada momento histórico. Sin embargo, las comunidades "primitivas" aportan al mundo de Occidente el valor de la solidaridad y la ética hacia el bien común que dan soporte a la eficiencia y eficacia que persiguen las empresas.

No sobra indicar que planear con base en la prospectiva en rupturas de estrategias en mercados deprimidos, se descubren sinergias derivadas de la solidaridad en equipos de trabajo, reconoce aportes del grupo en vez de resaltar los aciertos individuales en procesos de sostenimiento de estrategias que garantizan la permanencia de la empresa en entornos amenazantes. Es más, el ingenio humano siempre ha estado presente en todas las etapas del desarrollo socioeconómico y cultural.

No sobra advertir, que desde el periodo industrial la fragmentación de los recursos naturales, soporte físico de avances tecnológicos y acumulación de capital resultan antagónicos e irreconciliables, simplemente porque dejaron de existir en las mentes de individuos que promovieron grandes transformaciones económicas desde la empresa como unidad organizacional.

\section{Cibereconomía, Marketplaces en redes de internet}

Del periodo electrónico de las computadoras pasamos a nanotecnologías y la cibereconomía que deslocaliza procesos generados por la globalización. Internet redefinió lo privado, creó la ciudadanía global y otros fenómenos conocidos como e-commerce e e-business (Bula, 2011). Internet a diario sacude recónditos escondrijos de la naturaleza humana: allí nacen y se solazan las creencias, hábitos y representaciones inconscientes de lo privado, lo inaccesible, lo inenarrable. Desde este ángulo, los mismos protagonistas se cotejan a sí mismos al ventilar percepciones de intimidades y tabúes de origen sexual en formas inéditas de interacción humana, donde el otro es un extraño o un conocido que también simula realidades. En estos escenarios socioculturales de las redes sociales se instalan las estrategias de marketing en sinfín de productos condicionados por los tipos de personalidad de consumidores, cada vez más exigentes en la selección de productos.

El consumo en marketplaces o cibermercados conmina nuevos perfiles de líderes y adaptación rápida al cambio de estrategias gerenciales y opciones de permanencia ante aperturas económicas asimétricas, competencias excluyentes y despiadadas en mercados deprimidos. Si el fordismo se delimita en la fábrica con énfasis en el ethos del trabajo en la cadena de productos en serie, hoy en día el consumo se realiza en el ciberespacio. Bula (2011) afirma que "Las posibilidades de comprar por e-Bay, Mercadolibre.com, Deremate.com, por ejemplo, dependen para comenzar, de poseer tarjeta de crédito, y obviamente de contar con la capacidad de adquirir bienes o servicios allí ofertados. Esta estratificación genera nuevos tipos de ciudadanía" (p. 173).

Otra característica, Harvey (1990, citado por Bula, 2011) es la "compresión espacio-temporal en la forma en que se tejen las relaciones sociales" ( $p$. 174). Entre los consumidores se marcan las diferencias socioeconómicas ajustadas a las facilidades de acceso a diferentes formas de pago en globos de consumo sin importar el lugar del planeta. En última instancia, la intermediación de servicios bancarios a través del plástico demuestra la capacidad de pago y acceso a información, dejando entrever nuevas formas de estratificación social.

En este orden de ideas, las tecnologías de información y la comunicación (TIC) en diferentes espacios y el mismo tiempo -aunque existan diferencias de horario entre América Latina, la Unión Europea o el continente asiático por ejemplo-, los actores pueden realizar transacciones económicas, participar en campañas políticas 0 administrativas simultáneamente. Conviene señalar, que internet se enlaza con el neoliberalismo de 0ccidente para generar situaciones de riqueza extrema por un lado; mientras que por el otro, la pobreza, el desempleo y las subcontrataciones son el orden del día con notable 
Rasgos de la práctica de la administración en la civilización agrícola, la industrialización y la cibereconomía en mercados globales

deterioro de las condiciones laborales de millones de personas en el planeta.

\section{Cambio climático}

No sobra advertir el pronóstico del Programa para el desarrollo de las Naciones Unidas (PNUD) en Colombia, con respecto al impacto del cambio climático sobre todo en las condiciones de vida de los habitantes de la zona rural en las regiones colombianas, especialmente en territorios de explotaciones mineras y agronegocios con la siguiente advertencia:

Aumento de los eventos extremos, las inundaciones y tormentas, las olas de calor y las enfermedades transmisibles. El elemento común a todos los insumos del Grupo Intergubernamental de Expertos sobre el Cambio Climático (IPCC) es que generan preocupación acerca de la sostenibilidad de los recursos naturales y de los servicios ecológicos, en relación con los medios de vida de las poblaciones, especialmente de las más pobres. Ésta es la primera conexión clave entre pobreza y cambio climático. (2009, p. 9)

Agregando a lo anterior, Kotter y Cohen (2010 citado por Shiffman \& Lazar) consideran que el desafío principal de las mejores organizaciones está en el capital humano. Esta orientación sugiere a los gerentes considerar los siguientes problemas: el desempleo, la responsabilidad social, la ética, la diversificación de la fuerza de trabajo, aspectos demográficos, competencia global y resalta "desafíos todavía no identificados". A pesar de este listado de problemas del entorno empresarial y corporativo no mencionan el cambio climático. Esta concepción supone que si las organizaciones responden a las necesidades de la sociedad, todos resultan beneficiados.

Estas problemáticas están en algunas agendas de alta gerencia, hacen parte de constantes desafíos no solo por las condiciones biogeográficas sino por las exigencias de los habitantes del entorno de las mismas. En cualquier caso, los aportes de la literatura administrativa en empresas corporativas o en unidades estratégicas de negocios deben superarse desde la prospectiva. Por su parte, David (1997) considera que: "En cierto sentido, el proceso de administración estratégica es un intento de imitar lo que ocurre en la mente de una persona brillante e intuitiva que conoce el negocio" (p. 6).

A diferencia de la planeación estratégica tradicional, la visión del gerente local se centra en la razón del ser del presente, sabiendo que éste instante de la realidad o del tiempo, porta en su interior el pasado. En otros términos, es la construcción del futuro con base en la visión de la empresa. Así pues, el éxito de ayer no garantiza el de hoy (David, 1997).

Por último, Bijon (1992) recomienda el enfoque etnológico que orienta la comprensión de la naturaleza humana del cliente inmerso en las relaciones sociales de su entorno natural. Agrega: "No son cifras lo que necesita el espíritu innovador, sino comprender desde adentro los mecanismos con el fin de evaluar su movilidad" (p. 64), sobre todo en países del Sur atrapados en políticas neoliberales; en Colombia se han cerrado más de 20.000 empresas en cuatro años. Los empresarios alegan que "(...) están produciendo a media marcha. La razón, el mercado no da para más. Inclusive, muchos de ellos han optado por cerrar sus industrias o cambiar de actividad" (El Tiempo, 2014).

\section{Conclusión}

Recorrer fragmentos de historia de la práctica administrativa y de la antropología cultural en el trabajo de equipo y de grupo desde la división técnica y modos de producción, situados en las antípodas del desarrollo económico se deriva lo siguiente: El trabajo complejo de la comunidad Gouro aporta al de organizaciones de la cibereconomía formas de cooperación afianzadas en iniciativas propias, inmediatas y acopladas a los propósitos que exige la actividad económica en tiempo y espacio del territorio en la aldea. Cada quien se adapta al otro desde la motivación del bien colectivo.

Ahora bien, en 0ccidente, el desafío de las empresas en mercados globales se cimienta en el individualismo. Bijon (1992) afirma que en la cultura francesa no se encuentra al otro como persona; si las cosas salen mal, simplemente se le responsabiliza. Este comportamiento es un obstáculo en la comprensión de los mecanismos profundos de la empresa y del 
mercado. Igual sucede en las culturas de las regiones en Colombia. Paradójicamente, el sostenimiento de estrategias de ruptura requiere que tanto jefes de línea como empleados de base desarrollen actitudes, valores solidarios y eficientes como sucede en las comunidades "primitivas" donde el grupo actúa en consonancia con la estrategia diseñada y en estado de alerta enfrente de imprevistos por amenazas del entorno.

\section{Referencias Bibliográficas}

Anónimo. (1974). Les marxistes contra l'autogestion. Paris: Abexpress.

Barnard, Ch., (1938). Coordinación y autoridad. En Duncan, W., (Ed.), Las ideas y la práctica de la administración. Los principales desafíos de la era moderna. (pp. 171-191). México: Castillo Hnos., S.A. de C.V.

Bijon, C. (1992). Las estrategias de ruptura. Bogotá: Tercer mundo en coedición con ediciones Uniandes.

Bula, J.I. (2011). La cibereconomía: ¿espacio de la ciudadanía global o de una nueva jerarquización social? En Sanabria, F. (ed.), Vínculos virtuales (pp. 173-187). Bogotá, Colombia: Universidad Nacional de Colombia.

David, F. (1997). Conceptos de administración estratégica. México: Prentice-HLL Hispanoamericana, S.A.

Ferris, T. (1988). ¿Por qué la administración?. En Duncan, W. J., (Ed.), Las ideas y la práctica de la administración. Los principales desafios en la era moderna. (pp. 3-20). México: Castillo Hnos., S.A. de C.V.

Haber, S. (1964). ¿El desafío de la eficiencia: hacer bien las cosas?. En Duncan, W. J., (Ed.), Las ideas y la práctica de la administración. Los principales desafíos en la era moderna. (pp. 55-80). México: Castillo Hnos., S.A. de C.V.
Nullvalue. (2015, agosto, 8). Cerradas cerca de 20 mil empresas, en cuatro años. El Tiempo. Recuperado de http://www. eltiempo.com/archivo/documento/MAM-777358

Programa para el desarrollo de las Naciones Unidas en Colombia. PNUD. Diálogo nacional: lucha contra la pobreza y adaptación al cambio climático. Bogotá, 03 de agosto de 2009.

Shiffman, L., \& Lazar K., (2010). Comportamiento del consumidor. México: Prentice Hall.

Schwartz, P. (1999). La administración y los gerentes. En Duncan, W. J. (Ed.), Las ideas y la práctica de la administración. Los principales desafíos en la era moderna. (pp. 21-54). México: Castillo Hnos., S.A. de C.V.

Solano, D. (2007). La transversalidad y transectorialidad en el sector público. XX Concurso del CLAD sobre Reforma del Estado y Modernización de la Administración Pública. Caracas. Recuperado de siare.clad.org/fulltext/0056804.pdf

Tamayo. M. (1995). La investigación. Módulo 2. Cali: Publicaciones Icesi.

Terray, E. (1971). El marxismo ante las sociedades primitivas. Buenos Aires: Editorial Lozada S.A.

Toffer, A. (1981). La Tercera Ola. España: Plaza \& Janes.

Werter, W., \& Keith Davis. Administración de recursos humanos. México: Mac Graw Hill.

Verdugo, W. Unidep. (2010, 11 de noviembre). Principios de administración. Recuperado de http://es.slideshare.net/ wenceslao/principios-administracin. Slideshares. 